\title{
MATRIX METALLOPROTEINASE MMP-2 AND MMP-9 ACTIVITIES IN SEMINAL PLASMA
}

\author{
Nina P. Ayvazova, \\ Violeta S. Rilcheva, \\ Emiliana I. Konova, \\ Roumen G. Roussev', \\ Pavel I. Rashev ${ }^{2}$
}

MC Clinical Institute for Reproductive Medicine-Pleven

${ }^{1}$ Department of Anatomy, Histology, Cytology and Biology, Medical University - Pleven ${ }^{2}$ Institute of Biology and Immunology of Reproduction "Acad. Kiril Bratanov", Department of Immunobiology of Reproduction, Bulgarian Academy of Sciences

\section{Corresponding Author:}

Nina Ayvazova

MC Clinical Institute for Reproductive

Medicine 20, Skobelev Blvd.

Pleven, 5800

Bulgaria

e-mail:ayvazovanina@abv.bg

Received: September 29, 2016

Revision received: November 21, 2016 Accepted: December 20, 2016

\section{Summary}

Matrix metalloproteinases (MMPs) are a group of proteases containing $\mathrm{Zn}$ ions as a cofactor, which are involved in degrading of a large number of extracellular matrix proteins, and bioactive molecules. They also play a major role in processes such as cell proliferation, cell migration, differentiation and apoptosis. Very little is known about the expression and function of MMPs in the male reproductive tract. Occurrence of MMP-2 and MMP-9 activity in human seminal plasma has been previously reported but their origin and function are still not fully understood. The aim of this study was to examine the presence of MMP-2 and MMP-9 in normal and abnormal human sperm samples and find if any correlation existed between the levels of expression of MMPs and fertilization potential of the spermatozoa. Human sperm samples were examined for the presence of MMP-2 and MMP-9 by gel zymography and western blot analysis. A DNA fragmentation test was performed. The samples were divided into two groups - samples with normozoospermia and teratozoospermia. The gelatin zymography showed gelatinolytic bands with molecular weight 64 and $72 \mathrm{kDa}$ corresponding to active and inactive form of MMP-2. MMP-9 was not detected. The MMP-2 enzymatic activity appeared to be much higher in samples with compromised sperm morphology as compared to the normozoospermic samples. The mean DNA fragmentation index (DFI) of the group with teratozoospermia was relatively higher (22.16\%) and over the upper reference limits, compared to the normozoospermic group, in which it was within the normal range $(17.26 \%)$.

Key words: sperm quality parameters, infertility, DNA damage

\section{Introduction}

Matrix metalloproteinases (MMPs) are group of proteases, containing $\mathrm{Zn}$ ions as a cofactor. They are involved in the degrading of a large number of extracellular matrix proteins (bioactive molecules). They also play a major role in processes such as cell proliferation, cell migration, differentiation and apoptosis. Most MMPs are secreted from the cell in an inactive form (pro-MMP). Their activation requires the Cys- $\mathrm{Zn}^{+2}$ (cysteine switch) interactions and cleavage of the propeptide. Substantial evidence supports a general role for MMPs in the control of 
many aspects of reproductive function that require tissue remodeling and cell growth [1]. MMPs have been known to take part in various processes in female reproductive function [2,3]. However, very little is known about the expression and function of MMPs in the male reproductive tract. Previous studies have identified expression of matrix metalloproteinases in human seminal plasma [4, 5]. These enzymes have also been found in the human fetal testis [6]. However, the identity and the exact function of MMP in the male reproductive system is still unclear. MMPs have been thought to play a role in cell remodeling during spermatogenesis, including sperm differentiation and morphological modification [7]. Matrix metalloproteinases MMP-2 (72 kDa) and MMP-9 (92 kDa) are expressed in many reproductive organs [2]. A significant amount of MMP-2 and MMP-9 seems to originate from the accessory sex glands such as the prostate and the seminal vesicles [4]. It has also been found that MMP-2 and MMP-9 are also synthesized in the testis, mainly by the Sertoli cells as demonstrated by immunohistochemistry studies in rats $[6,8]$. MMP-2 was also detected in Sertoli cells culture [9]. The presence of MMP-2 and MMP-9 activity in human seminal plasma has been previously reported but still their origin and function remain to be elucidated.

The aim of this study was to examine the presence of MMP-2 and MMP-9 in normal and abnormal human sperm samples and find if any correlation exists between the levels of expression of MMPs and fertilization potential of the spermatozoa.

\section{Materials and Methods}

\section{Sperm Preparation}

Semen samples were collected from patients undergoing assisted reproduction $(\mathrm{n}=15)$. The semen parameters were evaluated using methods and reference values recommended by the World Health Organization (WHO) [10]. The following parameters were evaluated in each sample: concentration (lower reference limit $>15 \times 10^{6} / \mathrm{ml}$ ), motility (lower reference limit $>32 \%$ with progressive motility), morphology (lower reference limit $>4 \%$ normal forms). A DNA integrity test was performed for every patient. The semen samples were collected after 3 days of sexual abstinence, liquefied at $37^{\circ} \mathrm{C}$ for 30 minutes, evaluated and processed for separating the seminal plasma from the spermatozoa by centrifugation through a double density gradient (Origin, Denmark) at $1600 \mathrm{rpm}$ for 20 minutes. The supernatant was carefully aspirated into a $1.5 \mathrm{ml}$ Eppendorf tube and stored at $-20^{\circ} \mathrm{C}$ until further analysis.

Aliquots of $500 \mu \mathrm{l}$ liquefied sperm samples were stored in liquid nitrogen for further evaluation of DNA fragmentation index (DFI).

\section{Gelatin Zymography}

Matrix metalloproteinase activities in seminal plasma were detected by gelatin zymography. Ten 1 from seminal plasma were electrophoresed under non-reducing conditions in a $10 \%$ Sodium Dodecyl Sulphate Polyacrylamide Gel Electrophoresis (SDS-PAGE) containing 0.1\% gelatin as substrate. After electrophoresis, the gel was washed in renaturing buffer $2.5 \%$ Triton X100 ) with gentle agitation $2 \times 30$ minutes at room temperature and incubated for 18 hours at $37^{\circ} \mathrm{C}$ in zymography digestion buffer $(50 \mathrm{mM}$ Tris, $0.2 \mathrm{M}$ $\mathrm{NaCl}, 5 \mathrm{mM} \mathrm{CaCl}_{2}, 0.02 \% \mathrm{NaN}_{3}, \mathrm{pH} 7.6$ ). The gel was stained with $0.5 \%$ Coomassie blue R250 in $40 \%$ ethanol - $10 \%$ glacial acetic acid solution for 4 hours and destained in the same solution without Coomassie. The enzymatic activity was visualized as clear bands against a blue background. Densitometry was performed using ImageTool software.

\section{SDS-PAGE and Western immunoblot}

Human seminal plasma was separated electrophoretically in non-reducing conditions in HOEFER miniVE SYSTEM (Amersham Biosciences, USA). The samples were mixed with $2 \times$ Sample buffer and loaded ( $15 \mu 1$ each) on $10 \%$ polyacrylamyde gel. SDS separation conditions were as follows: $80 \mathrm{~V}$ for 20 minutes in the loading gel and $140 \mathrm{~V}$ for 3 hours in the resolving gel. The molecular weights of the protein fractions were determined using prestained molecular weight markers (Fermentas, Latvia).

Proteins were transferred to a nitrocellulose membrane (Millipore, USA) for 1 hour in semidry blotting system (Bio Rad), blocked with 3\% non-fat dry milk in Tris-buffered saline (TBS $150 \mathrm{mM} \mathrm{NaCl}, 10 \mathrm{mM}$ Tris-HCl, $\mathrm{pH}$ 7.6) for 2 hours at room temperature and incubated overnight at $4{ }^{\circ} \mathrm{C}$ with anti-human MMP-2 and anti-human MMP-9 polyclonal antibodies (Santa Cruz Biotechnology, 1:200) in blocking solution. Following extensive washing with TBS, the blots 
were incubated for 1 hour with goat anti-rabbit IgG conjugated with alkaline phosphatase (Sigma Co., USA) diluted 1:40 000 in blocking solution. After being rinsed as described above, the blots were treated with $4 \mathrm{nM}$ 5-bromo, 4chloro, 3-indolylphosphate (BCIP, Sigma) and $18 \mathrm{nM}$ 4-nitro blue tetrazolium chloride (NBT, Sigma) in alkaline phosphatase buffer $[100 \mathrm{mM}$ Tris- $\mathrm{HCl}$ (pH 9.6), $100 \mathrm{mM} \mathrm{NaCl}, 5 \mathrm{mM} \mathrm{MgCl}$ ]. The molecular mass of the separated proteins was estimated by comparison with mobility of prestained molecular mass markers (Fermentas, Latvia).

\section{Sperm DNA Fragmentation Test}

Aliquots of $500 \mu \mathrm{l}$ liquefied sperm samples were treated with acid detergent solution $(\mathrm{pH} 1.2)$ containing $0.1 \%$ Triton X-100, $0.15 \mathrm{~mol} / \mathrm{L} \mathrm{NaCl}$, and $0.08 \mathrm{~mol} / \mathrm{L} \mathrm{HCl}$ for 30 seconds and stained with $6 \mathrm{mg} / \mathrm{L}$ purified acridine orange (Sigma) in a phosphate-citrate buffer (pH 6.0) to be subjected to analysis by flow cytometry (FCM). Sperm chromatin damage was quantified by the FCM measurements of the metachromatic shift from green (native, double-stranded DNA) to red (denatured, single-stranded DNA) fluorescence and displayed as red versus green fluorescence intensity cytogram patterns. DFI is the ratio of the percentage of sperm showing an increased red fluorescence/total fluorescence intensity (red + green) [11].

\section{Statistical Analysis}

All the samples were processed in duplicates. Statistical analysis of the data was carried out by the Student's t-test for comparing the two groups. The data are presented as mean \pm standard deviation (mean $\pm \mathrm{SD}$ ). A p-value less than 0.05 was considered significant.

\section{Results}

\section{Semen Analysis}

The patients were divided into two groups according to the results from the semen analysis samples with normozoospermia and teratozoospermia. The two groups did not differ significantly in terms of sperm concentration and progressive motility, but morphologically vary considerably (Table 1).

Table 1. Semen characteristics

\begin{tabular}{llll}
\hline & $\begin{array}{l}\text { Normozoospermia } \\
\text { Morphology } \mathbf{>} \mathbf{4} \%\end{array}$ & $\begin{array}{l}\text { Teratozoospermia } \\
\text { Morphology }<\mathbf{4} \%\end{array}$ & p-value \\
\hline $\begin{array}{l}\text { Sperm concentration } \\
\text { (sperm cells/ml) }\end{array}$ & $61.5 \times 10^{6} \pm 32.5 \times 10$ & $57.5 \times 10{ }^{6} \pm 27.7 \times 10$ & NS \\
\hline Progressive motility (a+b\%) & $44.3 \pm 7.1$ & $39.4 \pm 5.2$ & NS \\
\hline DFI $(\%)$ & $17.26 \pm 6.04$ & $22.17 \pm 6.95$ & $\mathrm{NS}$ \\
\hline
\end{tabular}

\section{Gelatin Zymography}

Two bands with molecular weight 64 and $72 \mathrm{kDa}$ were displayed on the gelatin-zymography gel in both groups, corresponding respectively to active and inactive form of MMP-2, MMP-9 activity was not detected (Figure 1). The bands obtained by gel zymography were quantified by densitometric scanning. Intriguingly the MMP-2 enzymatic activity appeared to be much higher in samples with compromised sperm morphology compared to the normozoospermic samples, although significant difference between the groups was observed only in enzymatic activity of active MMP-2 (act-MMP-2; pro-MMP-2

\section{Immunoblot Analysis}

Western immunoblot was done by using specific anti-MMP-2 antibody to confirm that these bands (64 and $72 \mathrm{kDa}$ ) represent MMP-2 (Figure 3).

\section{Sperm DNA Integrity Results}

Sperm DNA fragmentation test was performed for each semen sample in terms to determine the fertility potential of the spermatozoa [12]. A threshold value of $20 \%$ DNA damage has been established for achieving pregnancy in vivo [13]. The measured DFI values did not differ significantly between the groups but they were relatively higher and over the upper reference limits in the group of the teratozoospermic 


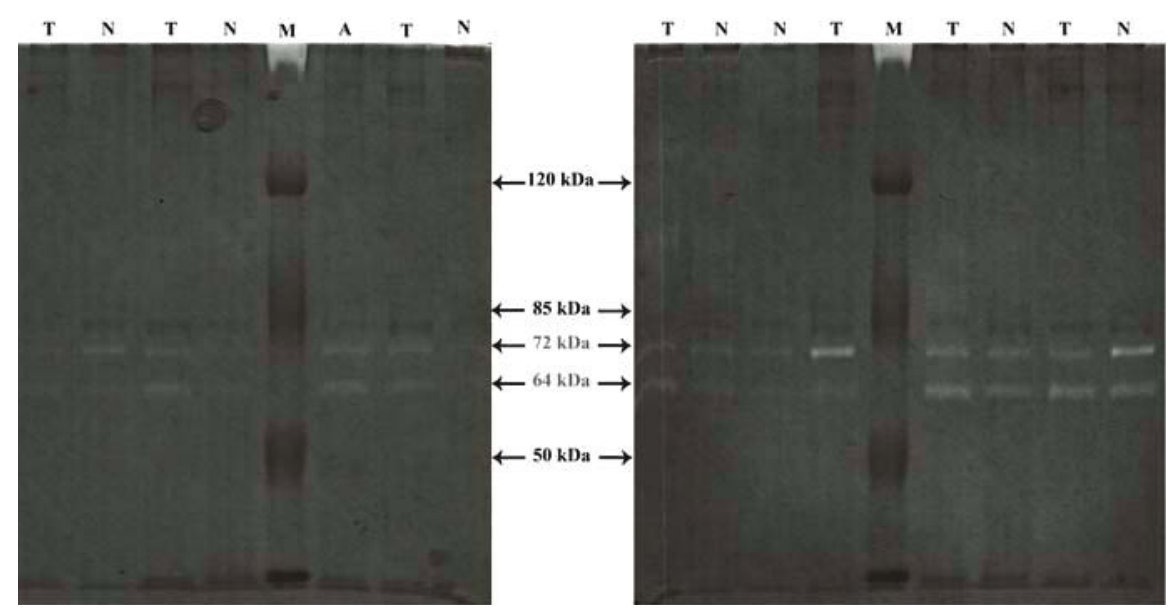

Figure 1. Gelatin zymography for detection of enzyme activity of matrix metalloproteinases in human seminal plasma from patients with Teratozoospermia (T) and Normozoospermia (N). Gelatinolytic bands with molecular weight 64 и $72 \mathrm{kDa}$ are visible. They represent the inactive and active form of MMP-2. MMP-9 was not detected

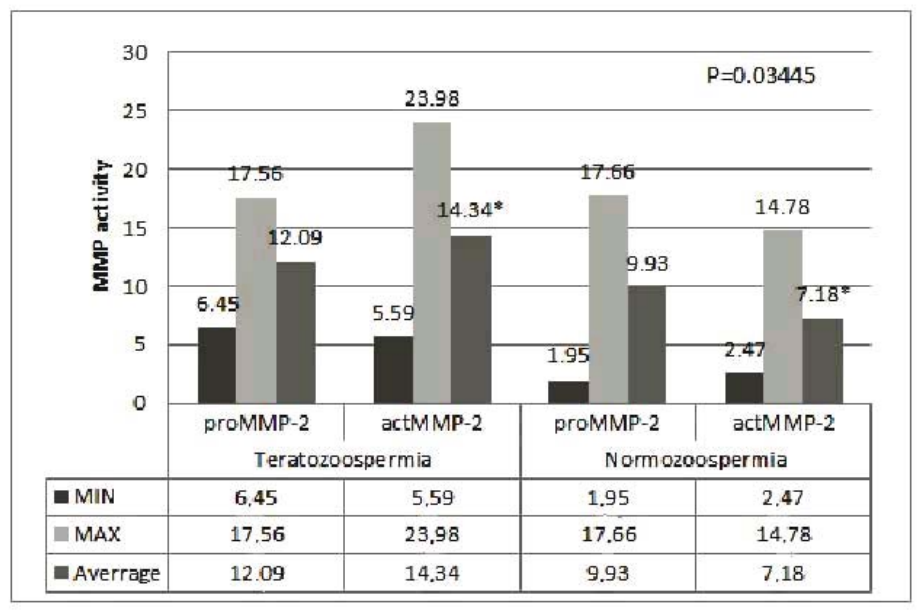

Figure 2. Densitometry analysis was used to measure the intensity of matrix metalloproteinase activity bands in gelatin zymography. Enzymatic activity of active form of MMP-2 was much higher in patients with pathological sperm morphology

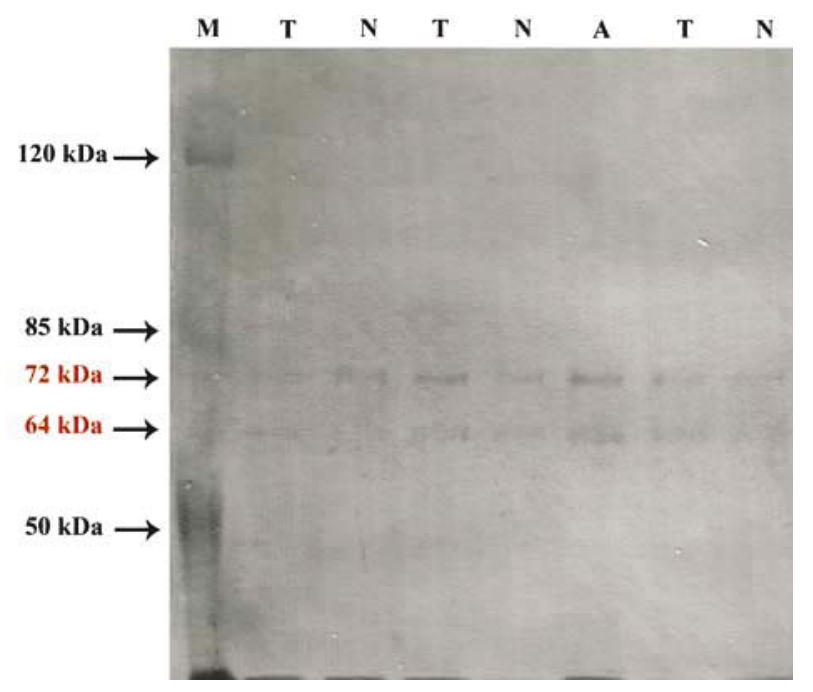

Figure 3. Western immunoblot was carried out for detection of MMP-2 in human seminal plasma from patients with Teratozoospermia (T) and Normozoospermia (N). By using specific antibody we confirm that these bands (64 and $72 \mathrm{kDa}$ ) represent MMP-2 
samples $(22.17 \pm 6.95 \%$, ranging from $12.2 \%$ to $32.3 \%$ ) compared to the normozoospermic samples $(17.26 \pm 6.04 \%$, ranging from $8.16 \%$ to $26.6 \%)$.

\section{Discussion}

Semen analysis and assessment of sperm DNA integrity continues to be the most reliable tool for the evaluation of male fertility potential, as sperm morphology is one of the most important parameters used for predicting the fertility potential in vivo [13]. The results from the current study revealed significantly increased activity of act-MMP-2 in semen samples with teratozoospermia. This is the first report that has found a correlation between MMP-2 activity and sperm morphology. Previous studies demonstrated that MMP-2 concentration in seminal plasma increased along with the increasing number of sperm in an ejaculate. However, no correlation was found between MMP-2 and sperm parameters such as motility or morphology [2, 3]. Meanwhile, other studies have indicated a negative correlation between metalloproteases (act-MMP-2 and pro-MMP-2) and sperm concentration, as well as motility. Considering the fact that semen samples included in the current study were with sperm count over $20 \times 10^{6} / \mathrm{ml}$ (Table 1 ), the activity of pro-MMP-2 and act-MMP-2 in the seminal plasmas we found is in agreement with the positive correlation between sperm concentration and MMP-2 activity established by some authors $[2,14]$. This could be explained by the fact that MMP-2 is usually not constitutively expressed but its expression can be enhanced or repressed to some extent. In addition, MMPs activity can be regulated post-transcriptionally by changes in mRNA stability by cleavage of the inactive form and interaction with inhibitors [15]. Probably this is the way that reproductive hormones and factors affect the MMP expression and concentration. In view of their biological activities, a potential role of MMPs in cellular proliferation, tissue growth and remodeling has been suggested. It is tempting to speculate that, in some cases, increased activity of MMPs in proliferative tissues could be involved in clearing basement membrane and/or connective tissue matrix components to make room for the multiplying cells to expand. Changes in the structure or integrity of the basement membrane may also alter the shape of the cell, modifying gene expression patterns and stimulating cell growth
[6].

In human seminal plasma, a higher concentration of pro-MMP-9 was detected in semen with abnormally low sperm concentration and vitality $[3,14,16]$. This could explain, to some extent, the absence of MMP-9 activity we observed in seminal plasmas with normal sperm count and motility corresponding to vital spermatozoa.

Other studies have indicated that higher concentrations of metalloproteases act-MMP-2 and pro-MMP-2 correlate with higher concentrations of acrosome damage [17]. This finding could be related to the fact that, in human sperm samples, MMP-2 and MMP-9 have been detected by immunofluorescence in the acrosome region and midpiece [3]. The acrosome reaction, i.e. the release of enzymes to penetrate the zona pellucida, should take place at the site of fertilization. Keeping these sperm proteases in an inactive form is crucial for maintaining cell integrity to ensure good reproductive function [18]. The elevated activity of act-MMP-2 and pro-MMP-2 observed in the teratozoospermic samples could be to some extent associated with increased acrosome damage and diminished fertilization potential.

The compromised sperm morphology is usually associated with impaired spermatogenesis. During spermatogenesis, a complex and dynamic process of proliferation and differentiation occurs as spermatogonia are transformed into mature spermatozoa. In this process, a series of mitoses and meioses occur, as well as changes in cytoplasmic content and structure, replacement of somatic cell-like histones with protamines, thus leading to a highly compacted chromatin structure [19]. MMPs located in basal lamina area may be involved in cleaving the protein components of the extracellular matrix or the cytoplasm of spermatozoa. MMPs activity might contribute to the remodeling of the basement membrane during the development of the seminiferous tubules and the subsequent release of differentiating spermatozoa [14]. Data from the current study showed a trend of elevated DNA damage in morphologically abnormal sperm samples, and such damage is not typical for normozoospermic ones. There is a strong correlation between the increased nuclear DNA damage in mature spermatozoa of men and poor semen parameters [20-22]. A number of studies have proposed that the prevailing presence in the semen samples of spermatozoa with damaged 
DNA is indicative of an initiated process of apoptosis. The mechanisms leading to abnormal spermatogenesis are poorly understood. This is possibly a result of impaired tissue homeostasis and this particular cellular population has, for some reason, escaped the programmed cell death and expression of various apoptotic markers the so called "abortive apoptosis" [23]. This phenomenon may be linked to defects in the cytoplasm remodeling during spermatogenesis and might be associated with the MMP-2 activity and function. The increase in DNA damage we detected in teratozoospermic samples is not statistically significant and needs further evaluation to find out if any correlation exists between increased DFI and MMP-2 activity and their possible role in the mechanism of sperm apoptosis. The final stage of sperm cells apoptosis is cell death [24, 25]. Studies performed with cardiomyocyte cell line have demonstrated a positive correlation between increase in the total MMP-2 and MMP-9 activities with cardiomyocyte apoptosis [26]. It has also been shown that pro-MMP-2 itself plays a role in the apoptotic pathway of neuron cells [27] and it is possible that MMPs might be involved in the initiation of sperm apoptosis via a pathway that is still undefined [14].

\section{Conclusions}

Our results demonstrate that MMP-2 activity correlates significantly with sperm morphology and is most probably connected with sperm fertilization potential. Therefore, the examination of metalloproteases in seminal plasma could be a valuable tool for semen evaluation and further research in this field is needed.

\section{Acknowledgements}

This work was supported by 7FP ReProForce project. Nina P. Ayvazova contributed with data collection and analysis, and wrote the paper. Violeta S. Rilcheva contributed to recruitment of patients, samples collection and storage and semen parameter analysis. Emiliana I. Konova contributed with experimental design and discussion of results. Roumen G. Roussev contributed to result discussion and experimental procedures. Pavel I. Rashev helped with the experiment design, experimental procedures and discussion of results.

\section{References}

1. Larsen M, Artym V, Green JA, Yamada KM. The matrix reorganized: extracellular matrix remodeling and integrin signaling. Curr Opin Cell Biol. 2006;18(5):463-71.

2. Hulboy DL, Rudolph LA, Matrisian LM. Matrix metalloproteinases as mediators of reproductive function. Mol Hum Reprod.1997;3(1):27-45.

3. Smith MF, Ricke W, Bakke LJ, Dow MPD, Smith GW. Ovarian tissue remodeling: role of matrix metalloproteinases and their inhibitors. Mol Cel Endocrinol. 2002;191(1):45-56.

4. Baumgart E, Lenk SV, Loening SA, Jung K. Quantitative differences in matrix metalloproteinase (MMP)-2, but not in MMP-9, tissue inhibitor of metalloproteinase (TIMP)-1 or TIMP-2, in seminal plasma of normozoospermic and azoospermic patients. Hum Reprod. 2002;17(11):2919-23.

5. Buchman-Shaked O, Kraiem Z, Gonen Y, Goldman S. Presence of matrix metalloproteinases and tissue inhibitor of matrix metalloproteinase in human sperm. J Androl. 2002;23(5):702-8.

6. Robinson LL, Sznajder N, Riley SC, Anderson R. Matrix metalloproteinases and tissue inhibitors of metalloproteinases in human fetal testis and ovary. Mol Hum Reprod. 2001;7(7):641-8.

7. Métayer S, Dacheux F, Dacheux JL, Gatti JL. Comparison, characterization, and identification of proteases and protease inhibitors in epididymal fluids of domestic mammals. Matrix metalloproteinases are major fluid gelatinases. Biol Reprod. 2002;66(5):1219-29.

8. Longin J, Guillaumot P, Chauvin M, Morera M, Le Magueresse-Battistoni B. MT1-MMP in rat testicular development and the control of Sertoli cell proMMP-2 activation. J Cell Sci. 2000;114(11):2125-34.

9. Qing-Xiang Sang, Dym M, Bryer SW. Secreted Metalloproteinases in Testicular Cell Culture. Biol Reprod. 1990;43:946-55.

10. World Health Organization. WHO laboratory manual for the Examination and processing of human semen. $5^{\text {th }}$ ed. Geneva: World Health Organization; 2010.

11. Lukanov T, Lichev D, Konova E, Emin A, Ayvazova N, Roussev G R, et al. Investigation of Sperm Dna Fragmentation By Sperm Dna Integrity Assay. J of IMAB. 2008;1:11-4.

12. Lukanov T, Lichev D, Konova E, Emin A, Ayvazova N, Roussev G R, et al. Flow cytometric measurement of sperm nuclear DNA fragmentation in infertile men with normal standard sperm parameters. J Mens Health. 2009;6(1):50-5.

13. Bungum M. Sperm DNA integrity assessment: a new tool in diagnosis and treatment of fertility. Obstet Gynecol Int. 2012;2012:1-6. 
14. Nikolettos N, Küpker W, Demirel C, Schöpper B, Blasig C, Sturm R, et al. Fertilization potential of spermatozoa with abnormal morphology. Hum Reprod. 1999;14(1):47-70.

15. Saengsoi W, Shia WY, Shyu C L, Wu JT, Warinrak $\mathrm{C}$, Lee WM, et al. Detection of matrix metalloproteinase (MMP)-2 and MMP-9 in canine seminal plasma. Anim Reprod Sci. 2011;127(12):114-9.

16. Matrbian LM. Metalloproteinases and their inhibitors in matrix remodeling. Trends Genet. 1990;6(4):121-5.

17. Tentes I, Asimakopoulos B, Mourvati E, Diedrich K, Al-Hasani S, Nikolettos N. Matrix metalloproteinase (MMP)-2 and MMP-9 in seminal plasma. J Assist Reprod Genet. 2007;24:278-81.

18. Pipan MZ, Kosec M, Mrkun J, Zrimšek P. Gelatinases in Boar Seminal Plasma and Their Relation to Semen Indicators. Acta Vet Brno. 2010;79(3):491-96.

19. Uhrin P, Dewerchin M, Hilpert M, Chrenek P, Schöfer C, Zechmeister-Machhart M,et al. Disruption of the protein $\mathrm{C}$ inhibitor gene results in impaired spermatogenesis and male infertility. J Clin Invest. 2000;106(12):1531-9.

20. Sakkas D, Moffatt O, Manicardi G C, Mariethoz E, Tarozzi N, Bizzaro D. Nature of DNA damage in ejaculated human spermatozoa and the possible involvement of apoptosis. Biol Reprod. 2002;66(4):1061-7.

21. Irvine D S, Twigg J P, Gordon E L, Fulton N., Milne P, Aitken R J. DNA integrity in human spermatozoa: relationships with semen quality. J Androl. 2000;21(1):33-44.
22. Lopes S, Sun J G, Jurisicova A, Meriano J, Casper R F. Sperm deoxyribonucleic acid fragmentation is increased in poor-quality semen samples and correlates with failed fertilization in intracytoplasmic sperm injection. Fertil Steril. 1998;69(3):528-32.

23. Sun JG, Jurisicova A, Casper R F. Detection of deoxyribonucleic acid fragmentation in human sperm: correlation with fertilization in vitro. Biol Reprod. 1997;56(3):602-7.

24. Sakkas D, Mariethoz E, St John JC. Abnormal sperm parameters in humans are indicative of an abortive apoptotic mechanism linked to the Fasmediated pathway. Exp Cell Res. 1999;251(2):350-5.

25. Aziz N, Said T, Paasch U, Agarwal A. The relationship between human sperm apoptosis, morphology and the sperm deformity index. Hum Reprod. 2007;22:1413-9.

26. Ricci G, Perticarari S, Fragonas E, Giolo E, Canova S, Pozzobon C, et al. Apoptosis in human sperm: its correlation with semen quality and the presence of leukocytes. Hum Reprod. 2002;17(10):2665-72.

27. Wohlschlaeger J, Stubbe H D, Schmitz K J, Kawaguchi N, Takeda A, Takeda N, et al. Roles of MMP-2/-9 in cardiac dysfunction during early multiple organ failure in an ovine animal model. Pathol Res Pract. 2005;201(12):809-17. 